\title{
LA MISERICORDIA DE DIOS EN EL ISLAM'
}

\author{
José Valdivia Valor \\ Doctor en Filología Semítica (Arabo-slámica) \\ Universidad de Alicante
}

\section{RESUMEN}

La Misericordia de Dios lo abarca todo. Los atributos de Dios referidos a su Misericordia y Munificencia vienen reflejados en los nombres Ar-Raḥmān y Ar-Rahịm. El primero es la cualidad exclusiva de Dios y se refiere a las gracias sobrenaturales y el segundo a las gracias naturales.

En cuanto al siervo del Misericordioso. 'Abd Ar-Raḥmān, resume este nombre y es una gracia para todo el mundo en general. 'Abd Ar-Rahìm, el siervo del Generoso, ejemplifica a la persona piadosa que practica la compasión pero sobre todo con aquellos en los que Dios se complace.

Palabras clave: Qư’àn, Tradición, Hadices, Misericordia, Ar-Raḥmān, Ar-Raḥīm.

\begin{abstract}
The Mercy of God includes all His creation. The Divine Qualities refered to the Compassionate and the Merciful are reflected in the names Ar-Raḥmān and Ar-Rahīm.

The first one is an exclusive quality of God and it is refered to the supernatural Grace and the second one to the natural Grace.

As for the servant of the Compassionate epitomises this name and is a mercy to all the world in general. 'Abd Ar-Rahìm, the servant of the Merciful exemplifies the pious that bestows his mercy particularly upon those with whom God is pleased.
\end{abstract}

Key words: Qur'ān, Tradition, Hadith, Mercy, Ar-Raḥmān, Ar-Raḥīm.

Tanto dentro del pensamiento islámico como del pensamiento cristiano podemos acercarnos a Dios por medio de sus atributos.

De entre los atributos que se le reconocen hemos elegido para nuestro estudio los que se refieren a su Misericordia Ar-Raḥmān y Ar-Raḥīm² . Para ello hemos recurrido tanto al Qur'ān

1 Mucho me complace esta oportunidad de poder participar en el Homenaje al profesor Joaquín Lomba Fuentes, nuestro querido amigo y colega que tan excelente labor ha realizado, sigue realizando, y que sea por muchos años, en la ciudad de Zaragoza y en su Universidad. Nunca olvidaré los buenos momentos pasados en nuestro primer encuentro en el año 1988 en la ciudad de Asilah con motivo del congreso sobre sufismo en Marruecos y AlAndalus amablemente invitados por el Ministro Mohamed Benaissa que tan cálidamente nos acogió junto a los demás componentes del congreso entre los cuales contábamos con los profesores M. Serguini, Makki y Rhermini..., entre otros. Sirvan para este Homenaje mi pequeña aportación y mi recuerdo para que siga manteniendo su buen ánimo y espíritu de trabajo.

2 Estos nombres junto a los demás atributos suelen utilizarse en repeticiones (dikr) para sentir la proximidad de Dios. Acerca del uso y práctica de estos nombres puede consultarse el artículo «Algunas consideraciones acerca de los nombres de Dios en el Islam. Beneficio que procura el uso de los mismos» J. Valdivia Valor Homenaje a María Jesús Rubiera en Shark Al-Andalus No 10-11, 1993-1994. Anales de la Universidad de Alicante pp. 683-692. 
como a algunos de los más destacados tratadistas, la mayoría de ellos místicos del Islam, que, desde antiguo, han venido ocupándose de este mismo tema. También nos hemos basado en el testimonio de los hadices o tradiciones que dan fe de los hechos y dichos de Muhammad el Profeta del Islam.

Suelen utilizarse en el Islam noventa y nueve atributos o nombres de Dios, atributos que, dicho sea de paso, ejercen una gran influencia en el pensamiento cristiano (a imitación de ellos reconoce el propio Lulio haber escrito sus «Noranta nou noms de Déu»).

Para empezar, podemos decir que, en el Islam, toda la creación ha sido realizada por Dios a causa de su Misericordia.

Vamos a referirnos en primer lugar a Ar-Raḥmān.

\section{AR-RAHMĀN}

Si nos atenemos al Qur'ān Dios dice: «Mi misericordia abarca todo», (Q.7:156). Dios quiere la Misericordia y lo bueno para toda la creación, en todo momento, sin distinción entre lo bueno y lo malo, el fiel y el rebelde, el amado y el aborrecido. Él derrama sobre la creación una munificencia infinita. La prueba está en el Qur'ān.

Cuenta el Sheik Tosum Bayrak en su tratado «De los más bellos nombres de Dios» que las personas que tienen conocimiento han interpretado el significado de Rahmān como el deseo que Dios tiene del bien absoluto, iradat al-jayr, y afirman que Raḥmān, así como Al-Lah, son los nombres apropiados para el Creador y no se pueden atribuir a nadie más. El significado de esta misericordia es la pureza de sentimiento, el dolor y la preocupación que se siente cuando sabemos que alguien está afligido. Pero el sentimiento de misericordia y compasión no es suficiente. La compasión real es eficaz cuando se es capaz de aliviar el sufrimiento y la pena de la persona afligida. Dios está por encima de esto. Sin embargo, antes de manifestar la creación optó por la compasión en vez de elegir el castigo. Él ha creado Su creación con Su misericordia. Todo lo que existe desde el comienzo ha sido bendecido con Su misericordia. Él ha creado todo, incluyendo Su creación suprema, el hombre, sin defecto y puro. Él ha bendecido su creación con infinita munificencia. En Su misericordia, Él ha mostrado los peligros de la perdición. Él ha dado al hombre y sólo al hombre la libertad de elegir entre el bien y el mal. Busca dentro de ti la luz de Rahmān — dice el Sheik-, usàndo tu libertad para escoger el bien para ti mismo y para los demás. Siente el sufrimiento de la persona mal guiada y también del infortunado, no condenándole sino compadeciéndole y ayudándole.

Y si nos atenemos a los hadices acerca de la Misericordia destaca entre estos uno de Abū Hurayra (Dios esté satisfecho de él) que nos informa sobre el Mensajero de Dios el cual dijo: «Dios el Altísimo tiene cien raciones de misericordia. Él ha enviado al universo una ración solamente y la ha dividido entre toda Su creación. El sentimiento de misericordia y compasión que Sus criaturas sienten entre sí no está dentro de esa porción. Las otras 99 porciones las ha reservado para el Día del Juicio Fina; ese Día Él las concederá a los creyentes».

Otro hadiz que refleja el deseo y la voluntad que tiene Dios de brindar su compasión y beneficencia a la creación es el siguiente: «Si uno no necesita pedir a Dios (Su compasión y Su beneficencia), Dios dirigirá su cólera contra él».

'Abd ar-Raḥmān es aquel en el que Dios manifiesta su misericordia en el universo. Cada hijo e hija de Hadrat Adán (Que Dios le bendiga) toma su parte de misericordia del Misericordioso según su potencial. Nadie ha quedado excluido de esta manifestación del Misericordioso. «Dios ha creadó al hombre a imagen de su Misericordia», dice en una tradición del Profeta (Que la paz y bendiciones sean sobre él) que es la misericordia de Dios en el universo ${ }^{3}$.

3 The Most Beautiful Names compiled by Sheikh Bayrak al-Jerrahi al-Halveti. Threshold Books. Amana Books. Vermmont 1985, pp. 21-22. 
Así en María, en Jesús, en Muhammad se ven reflejados en muy alto grado estos atribu$\operatorname{tos}^{4}$.

Veamos ahora lo que dice el místico sufí Muhy-dinn Ibn al-'Arabi de Murcia llamado el Sheij al-Akbar, Maestro Máximo, acerca de Ar-Raḥmān ${ }^{5}$.

En primer lugar, según él, este nombre indica dependencia, $t a$ 'alluq: Tienes necesidad iftiqār de este nombre para actualizar tah sill el nombre que de ti ignora el mundo de la creación 'alam al jalq sin que lo ignore el Mundo del Mandato 'alam al amr. En segundo lugar realización tahaqquq:

Refiere Ibn al-'Arabĩ que este nombre, como el nombre de Al-Lah, pertenece por su significado dalala a la categoría de los nombres propios privativos asmā'al-'a'lam. Por lo tanto puede recibir calificativos, pero no puede calificarse con él. Preguntaron los árabes coetáneos del Profeta al escuchar este nombre: «Y ¿qué es al-Raḥmān?» (Q.25:60), y al hacerlo expresaron su desconocimiento, pues si este vocablo lafza hubiera sido propio de su habla, deducible analógicamente por derivación etimológica ištiqãq, entonces no lo habrían ignorado; y si les hubiese resultado familiar y conocido como el nombre Al-Lah tampoco lo hubieran desconocido. En este sentido, cuando se les dijo: «!Adorad a Al-Lah! (Q.7:59)» no preguntaron qué era Allah, sino que, como es propio de los asociadores suraka u defensa: «No los adoramos (e. d., a los ídolos) sino para que nos aproximen a Al-Lah en grado (Q.39.3)». De manera que por esa razón el sabio murciano incluye este nombre en la categoría de los nombres propios, debido a que los árabes, como él mismo explica, no conocían esta palabra, al-Raḥmān, como nombre determinado, es decir, precedido del articulo al (alif-läm) aunque se tratase de un término derivado del nombre raḥma, (compasión).

Dice también que aquel que refuta la existencia del nombre al-Rahmān con el artículo [alif lām], considere no obstante que en el escrito que Salomón dirigió a Bilqis se dice: «En el nombre de Al-Lah, el Compasivo (al-Raḥmān), el Misericordioso (Q. 27:30)».

En definitiva, afirma que el artículo determina el significado del Nombre, y por ello no lo considera indeterminado. Aclara que toda su exposición se refiere exclusivamente a la palabra al-Raḥmān en la lengua árabe y que cuando el Profeta -Dios le bendiga y le salve- redactó el escrito entre él y los asociadores, escribió «En el Nombre de Dios, el Omnicompasivo (alRaḥmān), el Misericordioso», y los asociadores dijeron: «No conocemos a al-Raḥmān», y solamente estaban escribiendo en el nombre de Dios.

Y para que quede bien claro que se trata de un nombre perteneciente a la categoría de los nombres propios, lo confirma también con Su palabra, enaltecido sea, como está escrito en el Qur'ān: «Di: Invocad a Al-Lah o invocad a al-Raḥmān, a quienquiera que llaméis, Él posee los más bellos nombres» (Q. 17:110). De este modo, la divina revelación enseña que el significado madlül del nombre Al-Lah es idéntico al significado del nombre Ar-Rahmān, por lo cual dijo «Él posee..». y no «ellos dos tienen..». y, en consecuencia, la relación del siervo con respecto a este nombre, en cuanto a la realización, es igual a la relación que tiene con respecto al nombre de Al-Lah, que anteriormente ya lo había tratado.

4 Véanse los atributos de María en el Santo Rosario. También habla sobre María y sus atributos Daniel Gimanet «Les Noms Divins en Islam», Les editions du Cerf. Paris 1988. Sobre Jesús, véase Fray Luis de León «De los Nombres de Cristo», obras completas B.A.C. Madrid 1991 pp. 417-421: Sobre Muhammad véase la publicación de Mikel de Epalza «Los Nombres del Profeta en la Teología Musulmana» en «Encuentro», Documentos para el entendimiento Islamo-Cristiano, ${ }^{\circ}$ 59-60 marzo-abril, 1967.

5 El Secreto de los Nombres de Dios. Introducción, traducción y notas de Pablo Beneito. Editora Regional de Murcia. Colección Ibn al-'Arabī, Murcia 1996, pp. 37-48

Acerca de los atributos Ar-Rahmān y Ar-Rahīm véase tambiên The Presence of Superlative Compassion (Rahamut). On the Names al-Raḥmān al-Rahīm and Other Terms with the Lexical Root r-ḥ-m in the Work of Ibn 'Arabì de Pablo Beneito. Journal of the Muhyiddin Ibn 'Arabī Society Volume XXIV, 1998, pp. 53-86. 
En cuanto a la verificación del siervo tahaqquq al-'abd con respecto a este nombre, aspecto por el cual éste se distingue del nombre Al-Lah, consiste, según él, en que por vía de la Faz de la divina Realidad waŷh al-Haqq tenga conocimiento de un nombre relativo a lo que entre él y su Señor no puede llegar a conocer otro que Al-Lah -Enaltecido sea-, ya que si esto apareciera de modo manifiesto recaería sobre ello la negación inkār como ocurre en el caso del nombre Ar-Raḥmān.

Si tenemos en cuenta los conceptos de la época en que se desarrolla la vida de Ibn al'Arabī (S. XII) apreciaremos cómo el sabio murciano es un adelantado de su época —de hecho el místico es siempre una persona universal. A esté respecto veamos, por ejemplo, su pensamiento con respecto a la mujer. Lo observamos claramente en el tema acerca de los siervos de Dios o abdales a quien también llama sustitutos ${ }^{6}$.

Dice así: Se preguntó a uno de los gnósticos, «¿Cuántos son los sustitutos abdal?». «Cuarenta almas nafs» —respondió. Entonces se le preguntó: «¿Por qué no has dicho cuarenta hombres raŷul?» — «Porque entre ellos puede haber también mujeres nis $\bar{a}$ »». «Cada uno de ellos - sigue diciendo- desconoce el conocimiento particular del otro».

En tercer lugar el atributo Ar-Rahmmān indica adopción. La adopción de los rasgos de este nombre, dice, es igual a la adopción de los rasgos del nombre Al-Lah, salvo que este nombre, en tanto que nombre derivado, no pertenece a la categoría del nombre Al-Lah que no es un nombre derivado de ningún otro.

Corresponde a este nombre la gracia general rahma 'āmma que es la gracia de la existenciación rahmat al-iyad, según Su Palabra -Enaltecido sea-: «Y Mi gracia comprende todas las cosas» (Q.7:156), en la cual hace extensivo su alcance.

La compasión rahma procede de esta gracia universal en virtud de la cual los seres existentes mawyudat sienten en todo momento mutua simpatía y cada existente tiene compasión de sí mismo.

Para nuestro gran místico la adopción de los rasgos de este nombre conlleva que el siervo tenga compasión hacia todo lo que no es Al-Lah, sin distinción ni separación alguna, de modo que la universalidad umüm requiere de él, sin que ello dependa de ninguna imposición legal madamma šar'iyya. Añade el dicho de Abraham - la paz sea sobre él一: «He aprendido la generosidad karam de mi Señor». Y Al-Lah es el que concede el favor de la rectitud'.

\section{DE LOS NOMBRES DE DIOS Y LOS HADICES}

También es tradición en el Islam que venga acompañado el nombre de Dios de un Hadiz. Como en la obra Sumisión de Shems Friedlander en donde se relata junto a Ar-Rahmmān el siguiente hadiz: 'A'isha relató que Harith ibn Hishan le preguntó al Mensajero de Al-Lah: «¿Cómo te llega la Revelación?». «A veces llega como el tañido de una campana, y es lo más duro para mí, luego se marcha y recuerdo lo que fue dicho».

'A'isha dijo: «Le vi recibir la Revelación en un día extremadamente frío, y cuando esto cesó, de su frente caían gotas de transpiración». (Transmitido por Bukharī)

Este tañido puede corresponder al sonido de un teléfono o a un zumbido en el oído; al levantarse el auricular este sonido se detiene y se transmite el mensaje.

Los cambios físicos que afectaron al Profeta mientras recibía la Revelación no deben ser considerados epilepsia, como algunos se inclinan a creer; Él recibía estas palabras en un estado consciente y claro, como se verifica en el texto: «Yo retengo en mi memoria lo que ha sido dicho» ${ }^{8}$.

6 Ídem.

7 Ídem.

8 Submission. Sayings of the Prophet Muhammad. Selección de Hadices Shems Friedlander. Notas sobre Hadices Sheikh Muzaffereddin. WildWoodHouse, Londres 1977, p. 13. 
Pasemos ahora al segundo atributo de Dios, Ar-Rahīm

\section{AR-RAḤTM, LA FUENTE DE TODA BENEFICENCIA}

Él es la fuente de infinita misericordia y beneficencia, que recompensa con regalos eternos a los que usan Su munificencia y beneficencia para hacer el bien. Esto se menciona en el Qur'ān: «Él es compasivo y misericordioso (solamente) con los creyentes». (Q.XXXIII).

Ar-Rahīm significa beneficencia hacia los que tienen voluntad y libertad, y que las usan siguiendo el deseo de Dios y para Su complacencia. Cuando Dios dice: «He creado todo para vosotros..»., ésta es la expresión de su raḥmaniyya. Cuando encontramos esta munificencia oculta en todas las cosas, incluyéndonos a nosotros mismos, y cuando la usamos como Él quiere que lo hagamos, haciéndolo por Él, como se nos ha ordenado, se nos recompensa con la salvación eterna. Dios dice: «... y Yo os creé para Mí». Este gran honor es la expresión de su rậmaniyya.

Muyahid (Que Dios esté complacido con él) dijo: «Raḥmān pertenece a las personas de este mundo; Rahīm a las de la Otra Vida». Los que lo saben, rezan: Ya Raḥmãn ad-dunya waRahīm al-ajira- «jOh!, Raḥmān del mundo y Rahīm de la Otra Vida». Raḥmān es misericordia en los nafs, el ser del mundo. Rahịm es misericordia en el corazón. Rạ̣mān nos da el sustento en este mundo. Rahīm nos da la salvación eterna en la otra vida.

La manifestación de rahimiyya, en los creyentes, se muestra como un agradecimiento a Dios, que lo da todo. También se manifiesta como la capacidad de ser compasivo, de dar a los demás y de ocuparse de ellos, y esto también nos lo da Dios. La ausencia de orgullo cuando se es Su instrumento en las buenas acciones, darse cuenta de que Él es el Creador de los necesitados y de la satisfacción de sus necesidades, y extender la beneficencia de Dios a los que la necesitan, todo ello refleja rahmaniyya.

Si encuentras dificultades, ingratitud y resentimiento, debes soportarlo por Dios, porque recibirás recompensa aquí y diez veces más en la Otra Vida. No te vanaglories de tus buenas acciones, sobre todo delante de los que las reciben. Agradéceselo a ellos. Si no existiese su condición, no podrías ejercer tu compasión y generosidad.

Por otra parte, los que reciben compasión y cuidados deberían estar agradecidos a sus benefactores y recordarlos bien en todo momento, porque «El que no puede ser agradecido con el hombre no puede ser agradecido con Dios».

Pero no deben hacer dioses de sus benefactores, convirtiéndose en sus siervos, en vez de convertirse en siervos de Dios. Deben saber que el bien viene solamente de Dios; pero la herramienta que ha escogido es una herramienta hermosa, digna de respeto.

Los que encuentran en su ser el sabor de Raḥmān y Rahīm, atributos de Dios, y se acercan a su Creador a través de ellos, no pueden tener duda ni tristeza en sus corazones. Saben que, no importa lo que sùceda, Dios, ar-Rahmān, ar-Rahịm, tendrá misericordia de ellos, los salvará y los recompensará.

Los que piensan que la compasión de Dios, la misericordia y la beneficencia que aparecen en ellos son sus propias cualidades, haciéndose arrogantes, ciertamente se convertirán en los que dudan. Esa duda, en casos extremos, puede llevar al hombre a quitarse la vida.

'Abd ar-Rahīm es la persona piadosa, con un temor y amor a Dios constante. Su vida es un esfuerzo permanente para perfeccionarse según la prescripción del Islam, y es la persona con la que Dios está complacida. Es aquel que ha sido honrado con la manifestación de la compasión de Dios y Su beneficencia, que muestra hacia otros creyentes ${ }^{9}$.

9 The Most Beautiful Names compiled by Sheikh Bayrak al-Jerrahi al-Halveti. Threshold Books. Amama Books. Vermmont 1985, pp. 23-24. 
En cuanto a este atributo de Dios Ar-Rahīm, Veamos lo que dice el Sheij al-Akbar. Refiere éste igual que hemos visto al tratar de Ar.Rahman que Ar-Rahīm indica también, Dependencia ta'alluq: Dice que tenemos necesidad de este nombre para obtener la gracia especial rahma jassa que consiste en la felicidad eterna sa'ädat al-abad y explica de igual modo que este nombre indica realización tahaqquq. Dice que la Esencia divina requiere que haya en la existencia corrupción y salud, aunque la manifestación del Vengador no tiene prioridad alguna sobre la del Agraciador al-Mun 'im y que este nombre está relacionado con todo bien jayr que no conlleva implícito daño darar y con todo daño aparente en cuyo interior hay un bien.

También se vale del Qur'ān para la comprensión de este término: Y es posible entender que a este nombre corresponda Su palabra: «... y la destinaremos a aquellos que tienen temor reverente... (Q. 7:156)», según lo cual Al-Lah manifestó que esta gracia es limitada y está restringida muqayyada tras manifestar la absoluta incondicionalidad universal de la gracia general, de modo que su relación nisba respecto al siervo depende de esta limitación hadd.

Por último señala que este termino indica también adopción tajalluq y la adopción de los rasgos de este nombre consiste en la compasión del siervo (rahmat al- 'abd) con todo aquel de quien Ia divina Realidad al-Haqq le ha ordenado que se compadezca, aunque no con todos y en toda circunstancia, según se manifiesta en Su Palabra: «Por respeto a la ley de Dios, no uséis de mansedumbre $r a$ 'fa con ellos (Q. 24:2)», pues también se ha transmitido que «cuando el Profeta se encolerizaba a favor de la causa de Al-Lah, nada se resistía a su cólera» y que «AlLah manifestará cólera gadab el Día de la Resurrección» ${ }^{10}$.

Tambien el tratado «Sumisión» incluye, acompañando a ar-Raḥīm el siguiente hadiz:

El MENSAJERO DE AL-LAH (La paz y las bendiciones de Al-Lah sean sobre Él) ha dicho: «En el cuerpo, hay un pedazo de carne que cuando está sano, el cuerpo entero está sano y cuando se corrompe, el cuerpo entero está corrupto; es el corazón» ${ }^{11}$.

Dentro del pensamiento islámico, también Al-Gazzālī explica el significado de los noventa y nueve nombres de Dios contenidos en la tradición de Muḥammad, transmitida por Abu $\mathrm{Hu}-$ rarya. De Al-Lah dice que es el Nombre propio y exclusivo de Dios, que expresa su esencia en cuanto suma de todos sus atributos.

Los atributos Ar-Raḥmān y Ar-Rahīm significan para Al-Gazzālī la voluntad perfecta y universal de satisfacer todas las necesidades de dignos e indignos, tanto en ésta como en la otra vida. Hace la distinción entre Ar-Rạ̣mān, el Misericordioso, qụe es cualidad exclusiva de Dios y se refiere propiamente a las gracias sobrenaturales y Ar-Rahīm, el Compasivo, que hace referencia a las gracias naturales. Fruto de ambos nombres para el místico son la misericordia para con los pecadores y compasión para con los pobres y desgraciados. Llega con ello a la explicación de cómo se concilia la misericordia divina con la existencia del mal' ${ }^{12}$.

Para concluir diremos con 'Abd Al-Razzāq Al-Qāshāni que estos apelativos son igualmente referidos a los Siervos de Dios. Decía que El Siervo de Dios, 'Abd Al-Lah, es el hombre a quien la Verdad ha iluminado con todos sus Nombres, de manera que no hay devoto de la verdad que esté en un nivel más ensalzado. Realmente este nombre debería usarse solamente para el Profeta Muhammad y para el Polo de cada Era que sobreviene, de entre los herederos de su descendencia, aunque otros hayan sido llamados alegóricamente Siervos de Dios. La característica de cada uno de los nombres de Dios es tomada en conjunto en todos los nombres de Dios en virtud de la unicidad y la unidad de todos los nombres.

10 El Secreto de los Nombres de Dios, Ibn al-Arabī, Editora Regional de Murcia, 1966, pp. 47-48.

11 Submission. Sayings of the Prophet Muhammad. WildWoodHouse, Londres 1977.

12 Cfr. Miguel Asín Palacios, El justo medio en la creencia. Compendio de Teología dogmática de Algazel. Instituto de Valencia de Don Juan, Madrid, 1929, pp. 437-438. Llama AJ-Gazzālĩ esta parte de su tratado Libro del más sublime designio que explica el sentido de los bellísimos nombres de Dios. Explica en él los noventa y nueve nombres de Dios que D. Miguel Asín traduce y comenta. 
En cuanto al Siervo del Compasivo, 'Abd Ar-Raḥmān —dice Al Qāshāni- resume el nombre del Compasivo y es una gracia para todo el mundo en general. De esta manera nadie que tenga la capacidad para esto queda excluido de su compasión. El siervo del Misericordioso, "Abd Ar-Rahīm, ejemplifica el nombre del Misericordioso y otorga su merced especialmente al piadoso, al virtuoso y a aquellos en quienes Dios se complace. Pero es vengativo para aquéllos con quienes Dios está airado ${ }^{13}$.

Hemos basado nuestro estudio tanto en el Qur'ān como en la Tradición y el misticismo islámicos. De manera que así, como hemos citado al comienzo de esta charla la Aleya VII versículo 156: «Mi misericordia lo abarca todo», queremos mencionar tres nuevas citas tomadas literalmente del Qur'ān:

«Cuando vengan a ti los que creen en Nuestros signos, di: «Paz sobre vosotros!» Vuestro Señor Se ha prescrito la misericordia, de modo que si uno de vosotros obra mal por ignorancia, pero luego se arrepiente y enmienda... Él es indulgente, misericordioso». (Q. 6,54)

«Informa a Mis siervos de que Yo soy el Indulgente, el Misericordioso,..».. (Q. 15, 49) « ¿Creyentes! ¡Temed a Dios y creed en Su Enviado! Os dará, así, participación doble en Su misericordia, os pondrá una Luz que ilumine vuestra marcha y os perdonará. Dios es indulgente, misericordioso». (Q. 57, 28) ${ }^{14}$.

Finalmente para darle un carácter práctico a nuestro estudio, Dios nos perdone si en algún momento hemos caido en el olvido de Dios, de su Creación o de nuestros semejantes, recordemos que en todo momento en el Islam se utiliza la fórmula Bismi-l-Lah Ar-Rahmān ArRahīm, así comienza el propio Qur'ān: «En el nombre de Dios, el misericordioso, el clemente». Fórmula que se utiliza para iniciar todos los actos, al igual que se utiliza Al-ḥamdu-li-l-Lah, gracias a Dios o alabado sea Dios, al terminar una acción, si esto no se usa como mero formulismo, sino dicho y hecho con corazón.

Nosotros en la Tradición — como dice un maestro Sufí contemporáneo- pedimos la ayuda de los grandes Maestros del pasado, de nuestros santos, pedimos y buscamos la ayuda del Profeta, pedimos la ayuda, la guía y la misericordia de Dios...

«Uno se beneficia de la misericordia de Dios» ${ }^{15}$.

José Valdivia Valor

Filologías Integradas

Dpto. de Estudios Árabes e Islámicos

Universidad de Alicante E.03080

13 A Glossary of Sufi Technical Terms compiled by 'Abd al-Razzăq Al-Qāshāni. Texto árabe con traducción inglesa por el Dr. Nabil F. Safwat. The Octagon Press Ltd., Londres, 1991, pp. 91-92 ár/ 66 ingl.

14 El Corán, edición preparada por Julio Cortés, Editorial Herder, Barcelona, 1992, pp. 198, 320, 632.

15 Sayyed Omar Ali Shah, Conferencia pronunciada en Madrid en el Salón de actos del Hotel Cardenal el 3 de marzo de 1991. 